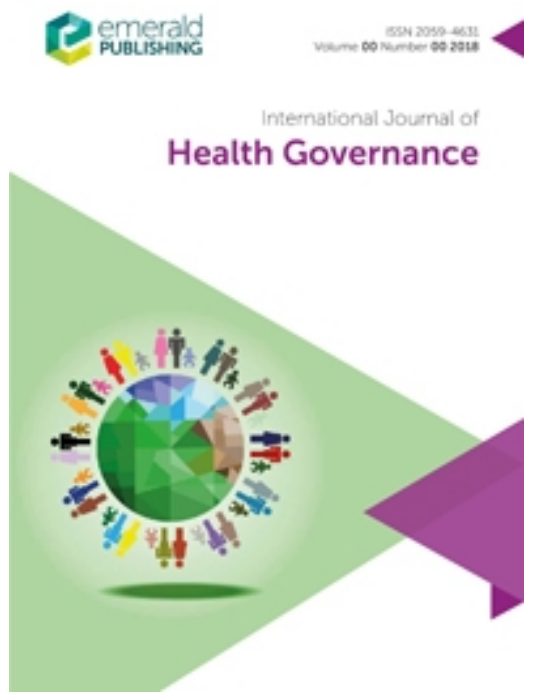

\title{
It takes a community to care for the sick and disabled
}

\begin{tabular}{|r|l|}
\hline Journal: & International Journal of Health Governance \\
\hline Manuscript ID & Draft \\
\hline Manuscript Type: & Clinical Governance Reviews \\
\hline Keywords: & $\begin{array}{l}\text { Primary care < Health Professions, Health Service Quality Assurance, } \\
\text { Clinical governance < Health Service Quality Assurance, Culture < Health } \\
\text { Service Quality Assurance, Patient perspectives < Health Service Quality } \\
\text { Assurance, Teamwork < Health Service Quality Assurance, User } \\
\text { involvement < Health Service Quality Assurance, Maternal \& child health }\end{array}$ \\
\hline \multicolumn{2}{|l}{} \\
\hline
\end{tabular}


IJHG 24.4

\section{It takes a community to care for the sick and disabled}

There is an old African proverb that states "It takes a village to raise a child" (Seymour 2013). This negates the modern Western model espoused by Bowlby (Bretherton 1992) of the mother as primary caregiver and attachment figure for the human infant. Indeed, examination of non-Western models of childcare and of other forms of caring demonstrate the importance of community involvement (AIFS 2014).

This review highjacks the traditional proverb and reimagines it to explore the importance of community engagement in healthcare. The review examines seven articles with varied perspectives from an exploration of Arabic language You Tube videos providing advice on cancer care through to a survey-based study that attempts to qualify how religious belief may affect empathy and action in volunteering with the sick or disabled. All of the articles included in this Special Issue have a common focus on community and collectively provide a lesson for healthcare in both the developing and the developed world that community plays an important role in ensuring essential, effective and equitable health care for all.

\section{Community in the internet age}

The first article reviewed conceptualized community somewhat differently than the way it is normally discussed in terms of healthcare. In traditional communities people ask advice of friends, relatives and neighbours. In seeking advice on cancer care, a worried individual might ask other people who have come through cancer treatment about what treatments worked for them, what the side effects of medication or radiography were, or if they had tried any alternative or complementary therapies. Today the internet offers new ways of becoming part of a community and this is particularly true in terms of health related topics. Ask your browser a question about health and you will soon find yourself connected to people willing to provide advice. Health forums can be opportunities to experience continuity of advice and support as McCarthy et al (2017) found in their study examining the use of Facebook forums as a focus for maternity care advice and support. However, members of the general public may find it difficult to discriminate between legitimate guidance based on current best evidence and untested theories derived from popular opinion. In McCarthy et al's study, Facebook forums were moderated by professional midwives who ensured the validity of advice shared between 'Facemums' as the women participating in the Facebook group were known. This level of governance was absent in the majority of You Tube videos analysed by Kharbat and Abu Daabes (2019) who selected You Tube as their medium because it is the $2^{\text {nd }}$ most visited 
social media site worldwide. They reviewed 150 videos in the Arabic language on the subject of cancer care. While 30 of these were later discarded due to lack of relevance, of the remaining 120 videos, $73 \%$ failed to mention risk factors or current recommendations concerning cancer treatment. The most visited videos were those uploaded by non-professionals and from analysing the questions and comments, the authors stated that it was clear that most viewers did not have the knowledge to differentiate between valid information and potentially dangerous misinformation. They concluded that closer governance of health related You Tube videos is needed.

\section{Conceptualising the wellness journey: First Nations communities in Australia and Canada}

Aboriginal and Torres Strait Islanders represent a more traditional picture of 'community'. First Nation Australians typically have very strong notions of community and attachment to their communities of origin (AIFS 2014). They also experience reduced life expectancy and higher rates of chronic health problems, compared to non-indigenous Australians (Rendall et al 2019). Yin (2017) suggests that much of this disparity, in Australia as in other post-colonial societies, can be attributed to historical trauma and racism. The tacit acknowledgement of these factors have led to increased effort on the part of the Australian government to increase community involvement in planning and participation in health care in indigenous communities. Government accreditation standards now require community engagement in decision-making around population health targets. A similar requirement to involve communities has existed in Canada for a number of years. As this requirement for community involvement has been maintained throughout the changing political scene in Canada and is now a well accepted construct of healthcare planning and provision, some lessons have been learned that may also be applicable to other communities such as the Australian Aboriginal and Torres island communities. These relate to how to effectively engage with communities and why this is important in healthcare planning and provision (Berland 2019). Clarity of purpose is essential together with good leadership and willingness to try different forms of engagement to meet a community's requirements. Berland (2019) suggest that recognition should be given for effort and not simply for outcomes when changes are being made within communities.

Rendall et al (2019) however point out the importance of differentiating between the community as a whole and consumers of health care who represent a subset of the wider community. The National Health and medical Research Council (NHMRC) of Australia has developed clear guidelines for community engagement. The central concepts underpinning this guidance are Building Relationships and Conceptualisation. The first of these is self-explanatory. If health professionals, including health researchers and care planners do not build relationships with the populations they serve then they are unlikely to develop a clear understanding a community's true needs. The second concept, Conceptualisation, relates to how people within a community visualize their 'wellness journey'. If what health providers offer is not congruent with what a community sees as its primary needs, communication and resolution will be complex and unproductive. Health services for indigenous populations must 
form a partnership based on respect and ethnographic knowledge in order to establish a service providing effective and culturally appropriate care.

The plight of elders trapped on college campuses designed for the young. Another article highlighting the need for culturally appropriate care is by Bhattacharyya, Chatterjee and Chopra Chatterjee (2019) who explain that traditional family structures and customs mean that many elderly people end up living with younger family members on academic campuses designed for the young. Not only do the campus health services focus on health needs of young people, the very design of campus life can be isolating for older residents. Many older family members are ineligible to use campus medical services even in emergency situations and as many academic campuses have been constructed in rural areas, it is difficult for older people to get to health services designed to meet their needs. While these problems may be exacerbated by campus life, they are by no means restricted to the campus environment. Bhan et al (2017) report that the ageing population is an increasing problem in a country lacking the health infrastructure to support their needs, particularly in rural areas. One issue raised by Bhan et al (2017) and by Bhattacharyya et al (2019) is that the burden of care for elderly people falls on family members, particularly female relatives. Often this will be a daughter-in-law as traditionally elderly parents choose to live with a son and his family (Bhattacharyya et al 2019). The changing structure of family life in India as elsewhere means more women are employed outside the home, often leaving elderly people feeling lonely or isolated. Greater community engagement for the elderly was one proposed solution (Bhattacharyya et al 2019; Bhan et al 2017), with Bhattacharyya et al (2019) reporting on elderly self-help initiatives including a yoga club, a micro-finance initiative and a hospital transport service. However, in order to prosper, such self-help programmes require support from the wider community and national government. Elderly people in India have traditionally been honoured for their life-experience and wisdom. Structuring India's healthcare to meet the chronic health needs of an ageing population would help elderly people to feel that they are still valued by society while supporting them to take on more active roles in meeting their own individual and collective requirements for health and social inclusion.

\section{Meeting the healthcare needs of disabled people in rural Nigeria.}

Elderly people are not the only members of a community who can have troubled accessing care. Zandam and Juni (2019) report on the problems of disabled people in rural Nigeria as they attempt to access appropriate acute and ongoing healthcare. The authors explain that a major issue is that the disabled lack basic rights afforded to other citizens. Caring for the disabled is considered the remit of charitable institutions and therefore public health services do not have a responsibility to ensure access and equity.

However, evaluating the experiences of people with disabilities, in terms of accessing health care serves as a good indicator of how well the service functions for the larger population. In other words, if the service works well for those who are most at a disadvantage in terms of their ability to access care, it works well for the larger population. Such an assessment also serves as a reminder that 
health is a vital component of human capital, enabling everyone to live a healthy and productive life, contributing to their communities to the best of their ability.

Zandam and Juni's (2019) research method was a population-based survey in the Jigawa state of Nigeria. 426 participants were evenly divided between disabled people and matched non-disabled counterparts.

The study examined the 'fit' between population requirements and current health service provisions using a model first developed by Pechansky and Thomas (1981) and subsequently adapted by other health researchers. The version used in this study employed 6 dimensions: availability, accessibility, affordability, acceptability, accommodation and approachability. The "6 As" approach was used to identify barriers to expressed needs.

Disabled participants had experienced impaired mobility for at least 6 months or had difficulty with a range of activities including walking, stair-climbing or self care. The access to healthcare study instrument was developed as a structured questionnaire that included demographic details and questions relating to the 6 dimensions of health care access.

Results showed that the disabled population was younger, more likely to live in rural areas and to be single. They also had lower levels of education, literacy and employment. $49 \%$ of the disabled belonged to the poorest economic group and only $28 \%$ considered themselves to be in good health, compared to $42 \%$ of nondisabled residents. The results were evaluated using a health accessibility index with the higher scores representing the most difficulty in accessing health care.

The areas having the most impact on disabled people's ability to access healthcare were (in order of importance) accessibility, affordability and location.

Findings demonstrated that disabled people experience high levels of healthcare need but also high levels of difficulty in meeting those needs. Some difficulties are related to the disability itself, but others are due to the perceptions of other people within their communities.

The authors propose changes to the health service, including financing,, decentralization of services, disability-friendly accommodation, and better communication on the part of healthcare workers to make health care more accessible and equitable for disabled people.

\section{Does religious belief increase empathy towards the ill or disabled?}

Zandam and Juni (2019) point out that in Nigeria, it is considered the role of charities, many of these run by religious organisations, to care for the disabled. While this is slowly changing to a more community-based approach, a team of researchers in Indonesia asked some interesting questions about whether religion makes people more likely to express empathy in terms of caring for the ill or disabled members of a community. Pratano et al (2019) became interested in the links between healthcare provision and faith groups, particularly in the charitable sector. In order to explore just how religiosity and empathy interact 
the researchers developed an online survey tool to test 4 hypotheses relating to these concepts. 610 respondents between the ages of 18 and 20 completed the survey. Findings seem to suggest that people identifying themselves as religious have less empathy but at the same time, more engagement with the health services in terms of volunteer activities. This lack of empathy may be due to stigma about illnesses associated with activities or lifestyles that conflict with the precepts of a certain faith, for instance sexually transmitted disease. At the same time there may be expectations for young people who identify as part of a faith community to express altruistic behaviour through activities such as volunteering in health care settings. Similar findings were reported by Watson, Hood and Morris (1985) who separated the concept of religiosity into 'intrinsic' and extrinsic'. Extrinsic religiosity was essentially self-serving and used religious adherence to create social acceptance or gain status or other individual goals. Intrinsic religiosity by contrast did lead to greater empathy and compassion as it was associated with deeply held beliefs.

Pratano et al (2019) conceptualized their study by starting with an interesting question and developing 4 hypotheses from this. They collected data using a research tool designed to be attractive to their target age group and they explained their analysis in detail. However, nuances are not revealed by statistics, thus it would be interesting to do a follow-up study using a more indepth qualitative methodology to examine how religion affects young people's attitudes and activities relating to illness and care provision.

\section{Can community engagement occur at a distance?}

In the final article reviewed Shroff (2019) asks whether partnerships between lower and middle income countries (LMIC) and countries in the Organisation for Economic Co-operation and Development (OECD) can improve maternal health outcomes, particularly in terms of a reduction in maternal mortality rates.

LMICs continue to have difficulty in reducing their maternal mortality rates despite efforts of the UN through implementation of the MDG programme. Shroff (2019) suggests that experts in OECD nations can offer help. Data collected through qualitative interviews with 10 global maternal health experts provided evidence that maternal health can be improved through better collaboration between interdisciplinary teams consisting of health professionals together with literacy teachers, agriculturalists and community development workers.

Using a community health model, the biggest improvements to maternal health are achieved through higher female literacy, gender justice, freedom from violence and food and water security as well as healthcare accessibility. Campbell, Calvert, Testa et al warn that all too often improving maternity care focuses on emergency responses. These researchers suggest that evaluation of routine care is just as necessary to evaluate providers' skills, prevent overintervention 
Shroff's recommendations include:

- Establishment of long term partnerships of 5-10 years between Canadian members and a region or nation in the Global South to improve women's health and reduce maternal mortality.

- Using Canadian midwifery graduates to transfer evidence based knowledge

- Provision of trained (midwives) attendants at all births

Maternity care is an excellent area in which to test such a partnership as health in pregnancy is the foundation for maternal and child health with implications throughout the life span (Steegers 2015). In addition, there are some existing forums for communication and collaboration between maternity care providers such as midwives and obstetricians from MLIC and HIC.

\section{Conclusion}

Raising children and raising awareness of the benefits of collaborative health care requires a change of attitude to a less individualistic, more holistic and community-based model. We CAN help each other to birth well, to live well and to die well. Today many traditional communities have become, or are becoming, lost. It is important to save old ways of being in communion with one another while we look for ways forge bonds for the future.

\section{References:}

Australian Institute of family Studies (AIFS) (2014) Strengths of Australian Aboriginal cultural practices in family life and child rearing. CFA Paper No. 25September 2014.

Accessed 08.09.19 from: https://aifs.gov.au/cfca/publications/strengthsaustralian-aboriginal-cultural-practices-fam/introduction

Berland A (2019) Lessons from the field for community engagement and accountability IJHG 24 (4):

Bhan N, Madhira P, Muralidharan A, Kulkarni B, Murthy GVS, Basu S, Kinra S (2017) Health needs, access to health care and perceptions of ageing in an urbanizing community in India: a qualitative study BMC Geriatrics 17. Accessed 11.09.2019 from: https://bmcgeriatr.biomedcentral.com/articles/10.1186/s12877-017-0544-y

Bhattacharyya T, Chatterjee C, Chopra Chatterjee S (2019) Health delivery system for older adults in academic campus in India IJHG 24 (4):

Bretherton I (1992) The origins of attachment theory: John Bowlby and Mary Ainsworth Developmental Psychology 25 (5): 759-775 
Campbell OMR, Calvert C, Testa A, Strehlow M, Benova L, Keyes E, Donnay F, Macleod D, Gabrysch S, Rong L, Ronsmans C, Sadruddin S, Koblinsky M, Bailey P (2016) The scale, scope, coverage and capability of childbirth care. The Lancet 388 (10056):2193-2208

Kharbat F, Abu Daabes A (2019) A content analysis of Arabic You Tube videos for cancer treatment IJHG 24(4):

McCarthy R, Choucri L, Ormandy P, Brettle A (2017) Midwifery continuity: the use of social media Midwifery 52: 34-41

Paradies Y (2016) Colonisation, racism and indigenous health. Journal of Population Research 33 (1): 83-96

Penchansky R, Thomas JW (1981) The concept of access: Definition and relationship to consumer satisfaction. Medical Care 19 (2): 127-140

Pratano A, Djoemadi F, Avanti C, Nur F, Maharani A (2019) Civic engagement in the Indonesia health sector, the role of religiosity, empathy and materialism attitude $I J H G 24(4)$ :

Rendalls S, Spigelman A, Goodwin C, Daniel N (2019) Health service engagement with consumers and community in Australia IJHG 24(4):

Seymour SC (2013) "It takes a village to raise a child": Attachment theory and multiple child care in Alor, Indonesia and in North India. In : Quinn N, Mageo JM (eds) Attachment Reconsidered. Culture, Mind and Society (The book series of the Society for Psychological Anthropology). Palgrave Macmillan, New York

Shroff F, Minhas J, Laugen C (2019) Power of partnerships: What makes a difference in reducing maternal mortality and how can Canadians contribute? IJHG 24(4):

Steegers E (2015)The public health importance of antenatal care OBGYN 7(1): 56

Watson PJ, Hood RW, Morris RJ (1985) Dimensions of religiosity and empathy Journal of Primary Care and Community Health (JPC) 4 (3): 73-85

Zandam H, Juni M (2019) Equity analysis of health system accessibility from perspective of people with disability IJHG 24(4): 